\title{
In vitro feasibility of next generation non-linear beamforming ultrasound methods to characterize and size kidney stones
}

\author{
Jaime E Tierney ${ }^{1}$, Siegfried G Schlunk ${ }^{1}$, Rebecca Jones ${ }^{1}$, Mark George ${ }^{1}$, Pranav Karve ${ }^{2}$, \\ Ravindra Duddu², Brett C Byram ${ }^{1,{ }^{*}}$, and Ryan S Hsi ${ }^{3,{ }^{*}}$ \\ ${ }^{1}$ Department of Biomedical Engineering, Vanderbilt University, Nashville, TN \\ ${ }^{2}$ Department of Civil and Environmental Engineering, Vanderbilt University, Nashville, TN \\ ${ }^{3}$ Department of Urologic Surgery, Vanderbilt University Medical Center, Nashville, TN
}

\section{Abstract}

Purpose-Ultrasound imaging for kidney stones suffers from poorer sensitivity, diminished specificity, and overestimation of stone size compared to computed tomography (CT). The purpose of this study was to demonstrate in vitro feasibility of novel ultrasound imaging methods comparing traditional B-mode to advanced beamforming techniques including plane wave synthetic focusing (PWSF), short-lag spatial coherence (SLSC) imaging, mid-lag spatial coherence (MLSC) imaging with incoherent compounding, and aperture domain model image reconstruction (ADMIRE).

Materials and Methods-The ultrasound techniques were evaluated using a research-based ultrasound system applied to an in vitro kidney stone model at $4 \mathrm{~cm}$ and $8 \mathrm{~cm}$ depths. Stone diameter sizing and stone contrast were compared among the different techniques. Analysis of variance was used to analyze the differences among group means, with $\mathrm{p}<0.05$ considered significant, and a Student's t-test was used to compare each method with B-mode, with $\mathrm{p}<0.0025$ considered significant.

Results-All stones were detectable with each method. MLSC performed best with stone sizing and stone contrast compared to B-mode. On average, B-mode sizing error \pm SD was $>1 \mathrm{~mm}$ $(1.2 \pm 1.1 \mathrm{~mm})$, while those for PWSF, ADMIRE, and MLSC were $<1 \mathrm{~mm}(-0.3 \pm 2.9 \mathrm{~mm}, 0.6 \pm 0.8$, $0.8 \pm 0.8$, respectively). Subjectively, MLSC appeared to suppress the entire background thus highlighting only the stone. The ADMIRE and SLSC techniques appeared to highlight the stone shadow relative to the background.

Conclusions-The detection and sizing of stones in vitro are feasible with advanced beamforming methods with ultrasound. Future work will include imaging stones at greater depths and evaluating the performance of these methods in human stone formers.

Corresponding Author: Ryan Hsi, Department of Urologic Surgery, Vanderbilt University Medical Center, A-1302 Medical Center North, Nashville, TN 37232, (615) 343-2036 (Phone), (615) 322-8990 (Fax), ryan.hsi@ vanderbilt.edu.

BCB and RSH contributed equally to this work as co-senior authors

ORCID: 0000-0003-1652-694X

Compliance with Ethical Standards

Ethnical Approval: This article does not contain any studies with human participants or animals performed by any of the authors.

Conflict of Interest: The authors report no competing conflict of interest disclosures. 


\section{Keywords}

ultrasonography; kidney; kidney calculi

\section{Introduction}

Kidney stones are highly and increasingly prevalent, and they are associated with significant morbidity, impaired quality of life, and health care utilization.[1-3] Management of stone disease is primarily dependent on diagnostic imaging to characterize stone burden, location, and associated urinary obstruction.

Computerized tomography (CT) is the gold standard imaging modality for kidney stone detection because of its high sensitivity and specificity for stone disease.[4,5] However, a major limitation of $\mathrm{CT}$ is the long-term cancer risk from ionizing radiation exposure,[6] which in stone disease is particularly relevant due to repeated imaging over the course of treatment and the increasing prevalence of stone disease among children and adolescents. $[7,8]$

Ultrasonography for kidney stone detection is portable, widely accessible, and avoids the concerns for malignancy risk. Additionally, ultrasonography provides information on the presence and degree of associated hydronephrosis that would suggest the presence of urinary tract obstruction. Despite these advantages, ultrasonography suffers from poorer sensitivity (24-69\%), diminished specificity (82-91\%), and overestimation of stone size of approximately $2-3 \mathrm{~mm}$ compared to CT. [9-14] This stone sizing error is significant because a rule-of-thumb threshold for size between observing stones versus recommending stone surgery is approximately $5 \mathrm{~mm}$. Based on this criterion for observation versus intervention, one study evaluating ultrasonography versus $\mathrm{CT}$ reported that $22 \%$ of patients could be inappropriately counseled when using ultrasonography alone. [15] Therefore, it is not surprising that in a randomized multicenter prospective trial comparing CT versus ultrasound as the initial screening test for acute renal colic, that among patients eventually needing intervention for kidney stones, $78 \%$ of all participants - whether they were in the CT or ultrasound arm - received CT imaging.[16] Therefore, ultrasound-based methods for stones that can overcome the detection and sizing limitations are needed. Several newer ultrasound imaging methods have been described, including detection of stone "twinkling" under Doppler mode[17,18] and stone shadow characterization for stone sizing[13]. However, drawbacks of these techniques include lower sensitivity for stones $<5 \mathrm{~mm}[19]$ and high false-negative detection rates.[20]

Our group has been investigating several novel ultrasound imaging methods using advanced beamforming techniques that may hold promise for improving ultrasound capability to characterize kidney stones. These include short-lag spatial coherence (SLSC) imaging, midlag spatial coherence (MLSC) imaging with incoherent compounding, aperture domain model image reconstruction (ADMIRE), and plane wave synthetic focusing (PWSF).[21-25] SLSC and ADMIRE are both non-linear ultrasound image formation methods that have both been shown to improve image quality. Specifically, both ADMIRE and SLSC belong to a small but growing set of ultrasound image formation methods that address the ubiquitous but 
understudied problem of reverberation and multipath scattering in clinical ultrasound.

ADMIRE is designed to retain characteristic B-Mode features in the process of improving image quality. In contrast, SLSC improves image quality by making images that are correlated to the spatial phase of the ultrasound wavefronts returning to the transducer, as compared to the traditional image formation techniques that are sensitive to the amplitude of the wavefronts. MLSC is designed specifically for improving ultrasound's sensitivity to calculi in general. MLSC aims to enhance coherent scatterers like stones, while suppressing the scattering that originates from soft tissue. In addition, we implement these methods in conjunction with synthetic aperture imaging; specifically, we use angled plane wave transmit beams, which we refer to as plane wave synthetic focusing (PWSF).[25]

These methods offer distinct strengths compared to traditional B-Mode imaging. In particular, ADMIRE and SLSC may enhance stone based features such as the shadow, and MLSC is designed to suppress incoherent sources of scattering (e.g. soft tissue) without suppressing stone scattering. Here, PWSF is used as a pre-processing step for all of the methods, but we also evaluate PWSF by itself. PWSF is the most directly comparable to BMode except that a transmit focus is synthesized at every point in the field. We hypothesize that the improvements and additional information provided by these techniques result in more accurate sizing and detection of stones. The purpose of this study is to demonstrate in vitro feasibility of PWSF, SLSC, MLSC, and ADMIRE for kidney stone characterization.

\section{Materials and Methods}

\section{Ultrasound imaging system}

A Verasonics Vantage 128 system (Verasonics, Inc., Redmond, WA) and L7-4 linear array transducer were used for ultrasound data acquisition (Figure 1A). The Vantage system is a flexible ultrasound system that contains acquisition hardware and an open, software-based research ultrasound platform. As a research based system, its design allows flexibility in defining the system's functional components that can be hard or impossible to implement using commercially available ultrasound systems.

\section{Experimental setup and imaging protocol}

Human calcium-based kidney stones ( $\mathrm{n}=12$, mean size $8.0 \mathrm{~mm}$, range $2-18 \mathrm{~mm}$ ) were used in this feasibility study. All stones were rehydrated and de-gassed at least $24 \mathrm{hrs}$ prior to imaging.

Stones were placed on top of gelatin phantoms while immersed in a water bath (Figure 1B). The gelatin phantoms were embedded with graphite to add diffuse scattering. The transducer was mounted above the stone and oriented to measure the maximum long axis length of the stone. Each stone was measured at $4 \mathrm{~cm}$ and $8 \mathrm{~cm}$ depths by varying the height of the submerged transducer. The stone position on the gel phantom was not changed when changing depths.

The raw channel data were recorded from angled plane wave transmissions ranging between $-30^{\circ}$ and $30^{\circ}$ spaced by $1^{\circ}$ using a center frequency of $5.2 \mathrm{MHz}$. The channel data were processed offline in MATLAB (Natick, MA). We assumed a sound speed of $1480 \mathrm{~m} / \mathrm{sec}$. 


\section{Ultrasound beamforming methods}

The raw channel data were processed using the following beamforming methods (Table 1 and Figure 2):

1. Conventional B-mode (B-mode)

Conventional beamforming applies delays to each transmitted channel signal to focus at a single depth and lateral location. The received signals are then dynamically delayed to achieve receive focusing at all depths for that lateral location. These channel signals are summed to generate a single line of the image. This process is repeated for multiple lateral locations to generate a full image. Here, the overall process has been also referred to as delay-and-sum beamforming (DAS).

2. Plane Wave Synthetic Focusing (PWSF)[25]

Transmit synthetic aperture focusing enables ultrasound systems to create synthetic transmit focuses throughout the image much like modern systems all utilize dynamic receive focusing to focus everywhere on the receive signal. Many different transmit synthetic aperture techniques are available, but here we implemented a scheme that relies on transmitting plane waves at different angles which we refer to as plane wave synthetic focusing (PWSF). It is akin to conventional B-mode imaging except that it achieves transmit focusing at all depths instead of at just a single depth.

3. Short-lag spatial coherence (SLSC)[21,22]

This method creates images correlated to the phase of the ultrasound wavefronts across the surface of the transducer. This phase can be calculated from the delayed and unsummed but transmit beamformed ultrasound channel data. The channel data are windowed to preserve axial resolution, and then each window is multiplied by the other channels and then normalized by the signal energy within the channels in order to create a measure of phase (i.e. coherence). The phase across nearby element spacings is summed to create each pixel in the final image. The original implementations of SLSC had a limited depth of field so we implemented SLSC with PWSF. SLSC has been shown to improve image quality in a range of scenarios, but is particularly suited to imaging difficult to image patients where image degradation is known to be a problem.

4. Mid-lag spatial coherence (MLSC) with incoherent compounding

This method is similar to SLSC but the order of the processing steps is rearranged to preferentially suppress the signal from tissue. To this end, MLSC performs the spatial phase quantification used with SLSC but before transmit beamforming. This means that there is no introduction of phase by the transmit beamforming as occurs in SLSC. Similar to SLSC, MLSC was implemented with PWSF. The phase images from each angled plane wave are summed together in order to enhance the stone and suppress any spurious correlations in the tissue. Additionally, because spurious points of coherence that may occur 
will occur in the shortest lags, these are excluded from the sum used to create the phase image; hence, the reference in the name to mid-lag.

5. Aperture domain model image reconstruction (ADMIRE) $[23,24]$

This technique is an explicit model-based method. The physics of linear ultrasound wave propagation are well known, and the effect of various sources of image degradation such as bright sources (i.e. stones) or reverberant sources such as those coming from shallow fat and muscle tissue can easily be modeled. By modeling a large number of these sources, the ultrasound wavefront returning to the transducer at a given time can be broken down into approximate points of origin. Then, ultrasound pressure waves originating only from a given region of interest can be reconstructed into a high-quality B-Mode signal. Like SLSC or MLSC, ADMIRE processing is applied to each pixel in the image and was implemented here with PWSF.

\section{Stone Contrast Characterization and Sizing}

After the data were processed via the respective algorithms to create each image type, the stone borders were identified using an automated segmentation algorithm implemented in Matlab.[26] This method iteratively assigns all pixels in the image to a class based on the intensity of the pixel and those surrounding it. This initial segmentation captures the entire stone, and minimal user input is then required to remove non-stone regions from the classification, which allows for greater consistency and accuracy compared to traditional sizing methods (e.g. manually) (Figure 3).

The contrast of the stone and shadow with respect to the visible gelatin background was calculated for each image to determine how visible the respective shadow or stone was relative to the surroundings. This measurement is independent of machine post-processing algorithms such as gain. The stone and shadow contrast values were calculated using the following formulas:

$$
\begin{gathered}
\text { contrast }_{\text {stone }}=20 * \log _{10} \frac{\mu_{\text {stone }}}{\mu_{\text {gel }}} \\
\text { contrast }_{\text {shadow }}=20 * \log _{10} \frac{\mu_{\text {shadow }}}{\mu_{\text {gel }}}
\end{gathered}
$$

where $\mu$ is the mean intensity of the stone, shadow, or gelatin background. Contrast values were negated as necessary so that higher values indicated that the stone or shadow is more different compared to the gel and vice versa.

Stone sizing was performed using the same automated segmentation technique noted above to calculate the lateral distance across the stone. 


\section{Statistical Analysis}

Accuracy of stone sizing was assessed by calculating the error (measurement error = ultrasound measured stone size - true stone size) for each measurement. Mean measurement errors were compared among the ultrasound methods at $4 \mathrm{~cm}$ depth, $8 \mathrm{~cm}$ depth, and overall. Similarly, stone and shadow contrast values were compared among the different methods. Analysis of variance was used to analyze the differences among group means, with $\mathrm{p}<0.05$ considered significant, and a Student's t-test was used to compare each method with Bmode, with a Bonferroni corrected significance level of $\mathrm{p}<0.0025$ due to multiple comparisons.

\section{Results}

All stones were detectable with each method. Stone sizing overall was best with MLSC at the $4 \mathrm{~cm}$ depth, but not at the $8 \mathrm{~cm}$ depth (Table 2, Figure 4 and 5). On average, B-mode sizing errors were $>1 \mathrm{~mm}$, while those for PWSF, ADMIRE, and MLSC were $<1 \mathrm{~mm}$.

Subjectively, MLSC appeared to suppress the entire background including the gelatin phantom. The ADMIRE and SLSC techniques appeared to highlight the stone relative to the shadow and gelatin phantom. The PWSF technique compared to B-mode appeared to suppress reverberation and clutter around the stone above the gelatin phantom.

Stone contrast relative to the gelatin phantom was highest with MLSC (Table 2, Figure 4 and 5). ADMIRE compared similarly to B-mode, while PWSF and SLSC had lower stone contrast than B-mode.

\section{Discussion}

There are several important findings in this feasibility study evaluating advanced beamforming methods for kidney stone characterization. MLSC compounding method appears to have promise in stone detection and sizing. It appears to highlight only the stone, while the adjacent standoff disappears. This is more than just "adjusting" the gain, because the background signals are almost completely suppressed. Furthermore, in this study, image quality did not qualitatively decrease with increasing depth, though this test was limited to only 4 and $8 \mathrm{~cm}$ depths. Each beamforming method appeared to enhance different properties of the stone. SLSC and ADMIRE both appear to highlight the shadow compared to B-mode alone. This is consistent with the previous descriptions of these techniques where they have been shown to suppress both off-axis and reverberation clutter, which likely decreases shadow visibility in many stones.[23,21,22] Further work will be needed to examine the shadow contrast and shadow width among these methods. In addition, ADMIRE and methods like SLSC and MLSC are known to be more robust to imaging environments encountered in the difficult-to-image patient. It is well understood that with increasing depths, the ability of ultrasound to detect and size stones deteriorates. Specifically, the lateral resolution increases linearly as a function of depth, and the signal-to-noise (SNR) decreases with depth. Patients with greater BMI, specifically skin-to-stone distances, are challenging with all imaging methods, including CT. So, despite our in vitro experiments conducted 
under idealized conditions, we expect that the relative improvements realized by these methods will actually increase in challenging patients.

These methods can be considered complementary to ongoing work to improve ultrasound performance for imaging kidney stones. Recent advances in stone characterization with ultrasound have included detection of stone "twinkling" under Doppler mode[17,18] and stone shadow characterization for stone sizing[13]. Using the color Doppler twinkling technique, sensitivity of detection is improved up to $>90 \%,[18,27,28]$ however the falsenegative rate is as high as $20 \%$ for renal stones.[20] The detection of the posterior stone shadow increases sensitivity and reduces sizing error, however for stones over $5 \mathrm{~mm}$, the shadow was visible $85 \%$ of the time, while under $5 \mathrm{~mm}$ it was visible only $53 \%$ of the time. May et al. have reported an algorithm to adjust gray-scale intensity with ultrasound to improve stone contrast and resolution using frequency $(4.5 \mathrm{MHz})$ and scanning line density (256 lines/frame), without using spatial compounding, speckle reduction, or other back-end processing.[19] They reported that compared to a clinical ultrasound system, this algorithm improved stone detection from $61 \%$ to $78 \%$.

To our knowledge this is the first study evaluating advanced beamforming methods for kidney stones. These methods are post-processing-type methods - that is, in principle, they do not require additional hardware over current commercial systems; although, in practice, only a subset of current systems may be able to implement the techniques proposed here. If these software methods were developed, matured, and validated, there is potential to backpack these on newer generation commercial imagers. Furthermore, the output of the ultrasound methods can easily be implemented within FDA limits for fundamental frequency ultrasound sequences. However, in the future it may be worthwhile to implement these techniques with tissue harmonic imaging. In this case it may be necessary to reconsider the transmit sequencing in order to remain within conventional FDA limits.

Future work studying these techniques will include broadening the parameters in the in vitro environment, including imaging stones at greater depths and embedding stones within tissue phantoms. These ultrasound methods are sensitive to stone roughness, and additional work is needed to explore the potential for determination of stone fragility and susceptibility to shockwave lithotripsy. Subsequent work will evaluate the performance of these methods in human stone formers and comparing the detection and sizing performance with clinical CT. Additional work is also needed to evaluate whether ureteral stones can be better visualized with these methods over B-mode, especially if no associated hydronephrosis is present

\section{Conclusions}

The detection and sizing of stones in vitro are feasible with advanced beamforming methods with ultrasound. MLSC appears to be promising as a sizing and detection method, while SLSC and ADMIRE enhance the contrast of the posterior acoustic shadow, potentially improving stone detection. Stone detection at limited depths did not appear to impact the performance of the methods. 


\section{Acknowledgments}

Funding: This study was funded by the Vanderbilt Institute of Surgery and Engineering (VISE) Pilot and Feasibility Award, VISE Surgeon in Residence Award, and R01EB020040.

\section{References}

1. Scales CD Jr, Smith AC, Hanley JM, Saigal CS. Urologic Diseases in America P. 2012; Prevalence of kidney stones in the United States. Eur Urol. 62(1):160-165. DOI: 10.1016/j.eururo.2012.03.052 [PubMed: 22498635]

2. Bryant M, Angell J, Tu H, Goodman M, Pattaras J, Ogan K. 2012; Health related quality of life for stone formers. J Urol. 188(2):436-440. DOI: 10.1016/j.juro.2012.04.015 [PubMed: 22704108]

3. Pearle MS, Calhoun EA, Curhan GC. Urologic Diseases of America P. 2005; Urologic diseases in America project: urolithiasis. J Urol. 173(3):848-857. DOI: 10.1097/01.ju.0000152082.14384.d7 [PubMed: 15711292]

4. Smith RC, Varanelli M. 2000; Diagnosis and management of acute ureterolithiasis: CT is truth. AJR American journal of roentgenology. 175(1):3-6. [PubMed: 10882237]

5. Smith RC, Verga M, McCarthy S, Rosenfield AT. 1996; Diagnosis of acute flank pain: value of unenhanced helical CT. AJR American journal of roentgenology. 166(1):97-101. [PubMed: 8571915]

6. Brenner DJ, Hall EJ. 2007; Computed tomography--an increasing source of radiation exposure. N Engl J Med. 357(22):2277-2284. DOI: 10.1056/NEJMra072149 [PubMed: 18046031]

7. Ferrandino MN, Bagrodia A, Pierre SA, Scales CD Jr, Rampersaud E, Pearle MS, Preminger GM. 2009; Radiation exposure in the acute and short-term management of urolithiasis at 2 academic centers. J Urol. 181(2):668-672. DOI: 10.1016/j.juro.2008.10.012 [PubMed: 19100573]

8. Tasian GE, Ross ME, Song L, Sas DJ, Keren R, Denburg MR, Chu DI, Copelovitch L, Saigal CS, Furth SL. 2016; Annual Incidence of Nephrolithiasis among Children and Adults in South Carolina from 1997 to 2012. Clin J Am Soc Nephrol. 11(3):488-496. DOI: 10.2215/CJN.07610715 [PubMed: 26769765]

9. Ulusan S, Koc Z, Tokmak N. 2007; Accuracy of sonography for detecting renal stone: comparison with CT. Journal of clinical ultrasound : JCU. 35(5):256-261. DOI: 10.1002/jcu.20347 [PubMed: 17373690]

10. Unal D, Yeni E, Karaoglanoglu M, Verit A, Karatas OF. 2003; Can conventional examinations contribute to the diagnostic power of unenhanced helical computed tomography in urolithiasis? Urologia internationalis. 70(1):31-35. DOI: 10.1159/000067702 [PubMed: 12566812]

11. Ray AA, Ghiculete D, Pace KT, Honey RJ. 2010; Limitations to ultrasound in the detection and measurement of urinary tract calculi. Urology. 76(2):295-300. DOI: 10.1016/j.urology. 2009.12.015 [PubMed: 20206970]

12. Fowler KA, Locken JA, Duchesne JH, Williamson MR. 2002; US for detecting renal calculi with nonenhanced CT as a reference standard. Radiology. 222(1):109-113. [PubMed: 11756713]

13. Dunmire B, Lee FC, Hsi RS, Cunitz BW, Paun M, Bailey MR, Sorensen MD, Harper JD. 2015; Tools to improve the accuracy of kidney stone sizing with ultrasound. J Endourol. 29(2):147-152. DOI: 10.1089/end.2014.0332 [PubMed: 25105243]

14. Sternberg KM, Eisner B, Larson T, Hernandez N, Han J, Pais VM. 2016; Ultrasonography Significantly Overestimates Stone Size When Compared to Low-dose, Noncontrast Computed Tomography. Urology. 95:67-71. DOI: 10.1016/j.urology.2016.06.002 [PubMed: 27289025]

15. Ganesan V, De S, Greene D, Torricelli FC, Monga M. 2017; Accuracy of ultrasonography for renal stone detection and size determination: is it good enough for management decisions? BJU international. 119(3):464-469. DOI: 10.1111/bju.13605 [PubMed: 27459091]

16. Metzler IS, Smith-Bindman R, Moghadassi M, Wang RC, Stoller ML, Chi T. 2017; Emergency Department Imaging Modality Effect on Surgical Management of Nephrolithiasis: A Multicenter, Randomized Clinical Trial. The Journal of urology. 197(3 Pt 1):710-714. DOI: 10.1016/j.juro. 2016.09.122 [PubMed: 27773846] 
17. Shabana W, Bude RO, Rubin JM. 2009; Comparison between color Doppler twinkling artifact and acoustic shadowing for renal calculus detection: an in vitro study. Ultrasound Med Biol. 35(2): 339-350. DOI: 10.1016/j.ultrasmedbio.2008.09.023 [PubMed: 19041171]

18. Abdel-Gawad M, Kadasne RD, Elsobky E, Ali-El-Dein B, Monga M. 2016; A Prospective Comparative Study of Color Doppler Ultrasound with Twinkling and Noncontrast Computerized Tomography for the Evaluation of Acute Renal Colic. J Urol. 196(3):757-762. DOI: 10.1016/ j.juro.2016.03.175 [PubMed: 27063853]

19. May PC, Haider Y, Dunmire B, Cunitz BW, Thiel J, Liu Z, Bruce M, Bailey MR, Sorensen MD, Harper JD. 2016; Stone-Mode Ultrasound for Determining Renal Stone Size. J Endourol. 30(9): 958-962. DOI: 10.1089/end.2016.0341 [PubMed: 27393000]

20. Lee JY, Kim SH, Cho JY, Han D. 2001; Color and power Doppler twinkling artifacts from urinary stones: clinical observations and phantom studies. AJR Am J Roentgenol. 176(6):1441-1445. DOI: 10.2214/ajr.176.6.1761441 [PubMed: 11373210]

21. Lediju MA, Trahey GE, Byram BC, Dahl JJ. 2011; Short-lag spatial coherence of backscattered echoes: imaging characteristics. IEEE Trans Ultrason Ferroelectr Freq Control. 58(7):1377-1388. DOI: 10.1109/TUFFC.2011.1957 [PubMed: 21768022]

22. Bottenus N, Byram BC, Dahl JJ, Trahey GE. 2013; Synthetic aperture focusing for short-lag spatial coherence imaging. IEEE Trans Ultrason Ferroelectr Freq Control. 60(9):1816-1826. DOI: 10.1109/TUFFC.2013.2768 [PubMed: 24658715]

23. Byram B, Dei K, Tierney J, Dumont D. 2015; A model and regularization scheme for ultrasonic beamforming clutter reduction. IEEE Trans Ultrason Ferroelectr Freq Control. 62(11):1913-1927. DOI: 10.1109/TUFFC.2015.007004 [PubMed: 26559622]

24. Byram B, Jakovljevic M. 2014; Ultrasonic multipath and beamforming clutter reduction: a chirp model approach. IEEE Trans Ultrason Ferroelectr Freq Control. 61(3):428-440. DOI: 10.1109/ TUFFC.2014.2928 [PubMed: 24569248]

25. Montaldo G, Tanter M, Bercoff J, Benech N, Fink M. 2009; Coherent plane-wave compounding for very high frame rate ultrasonography and transient elastography. IEEE Trans Ultrason Ferroelectr Freq Control. 56(3):489-506. DOI: 10.1109/TUFFC.2009.1067 [PubMed: 19411209]

26. Aja-Fernandez S, Curiale AH, Vegas-Sanchez-Ferrero G. 2015; A local fuzzy thresholding methodology for multiregion image segmentation. Knowl-Based Syst. 83:1-12. DOI: 10.1016/ j.knosys.2015.02.029

27. Ripolles T, Martinez-Perez MJ, Vizuete J, Miralles S, Delgado F, Pastor-Navarro T. 2013; Sonographic diagnosis of symptomatic ureteral calculi: usefulness of the twinkling artifact. Abdom Imaging. 38(4):863-869. DOI: 10.1007/s00261-012-9946-7 [PubMed: 23011549]

28. Kielar AZ, Shabana W, Vakili M, Rubin J. 2012; Prospective evaluation of Doppler sonography to detect the twinkling artifact versus unenhanced computed tomography for identifying urinary tract calculi. J Ultrasound Med. 31(10):1619-1625. [PubMed: 23011625] 

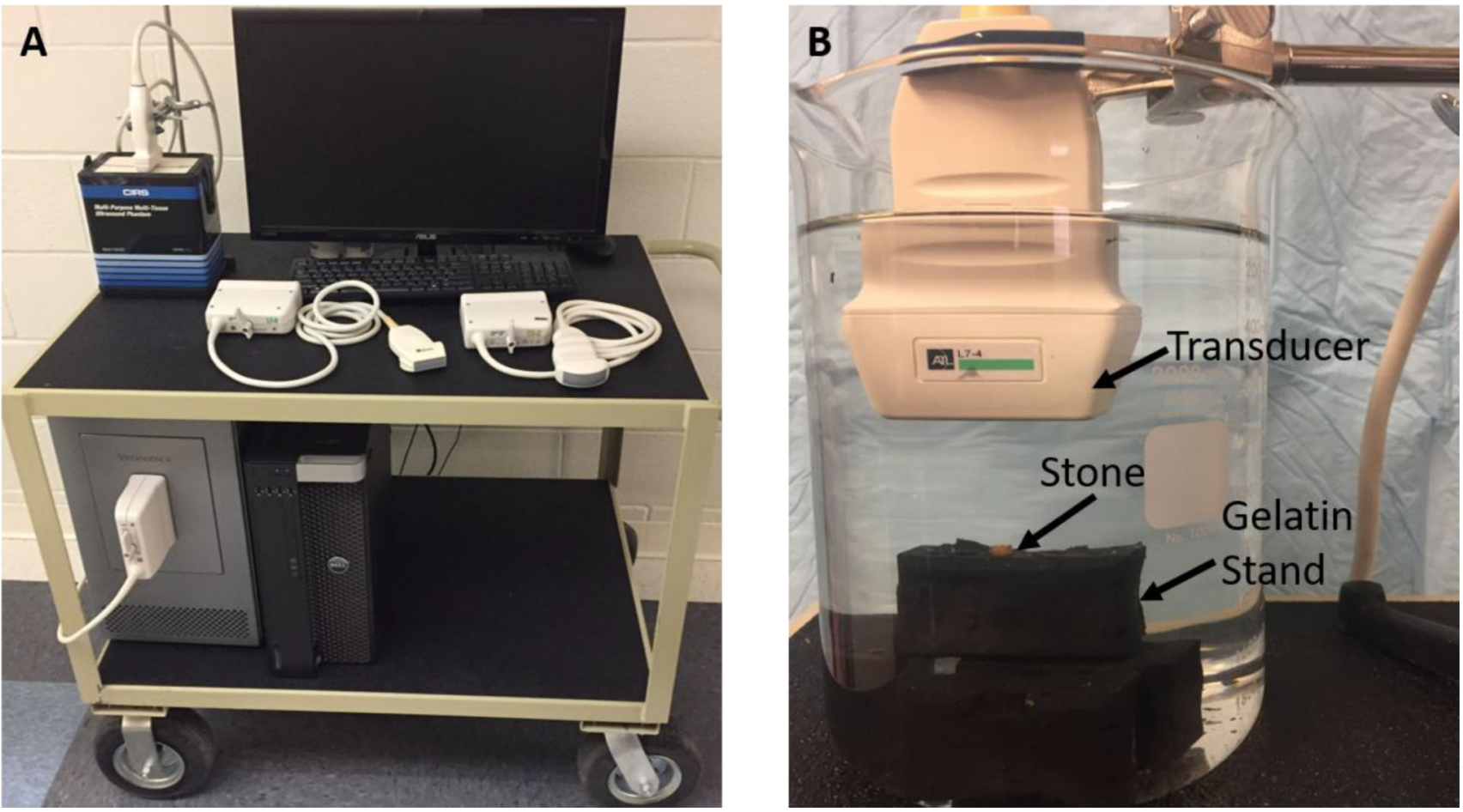

Figure 1.

(A) Verasonics Vantage 128 imaging system. (B) Experimental set-up: water bath with gelatin phantom and kidney stone. 


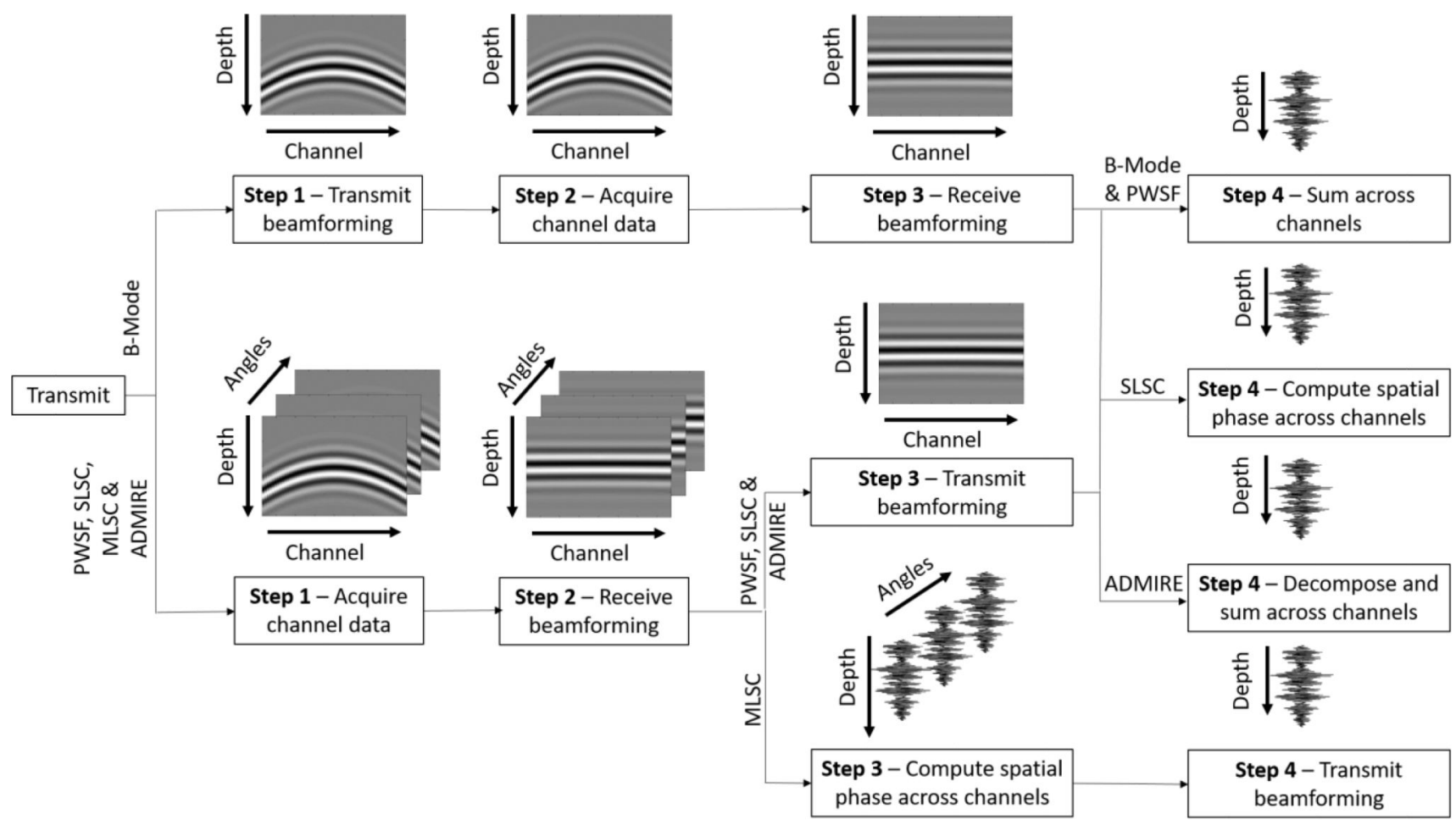

Figure 2.

Schematic of generating a single line of an image for each beamforming method. 


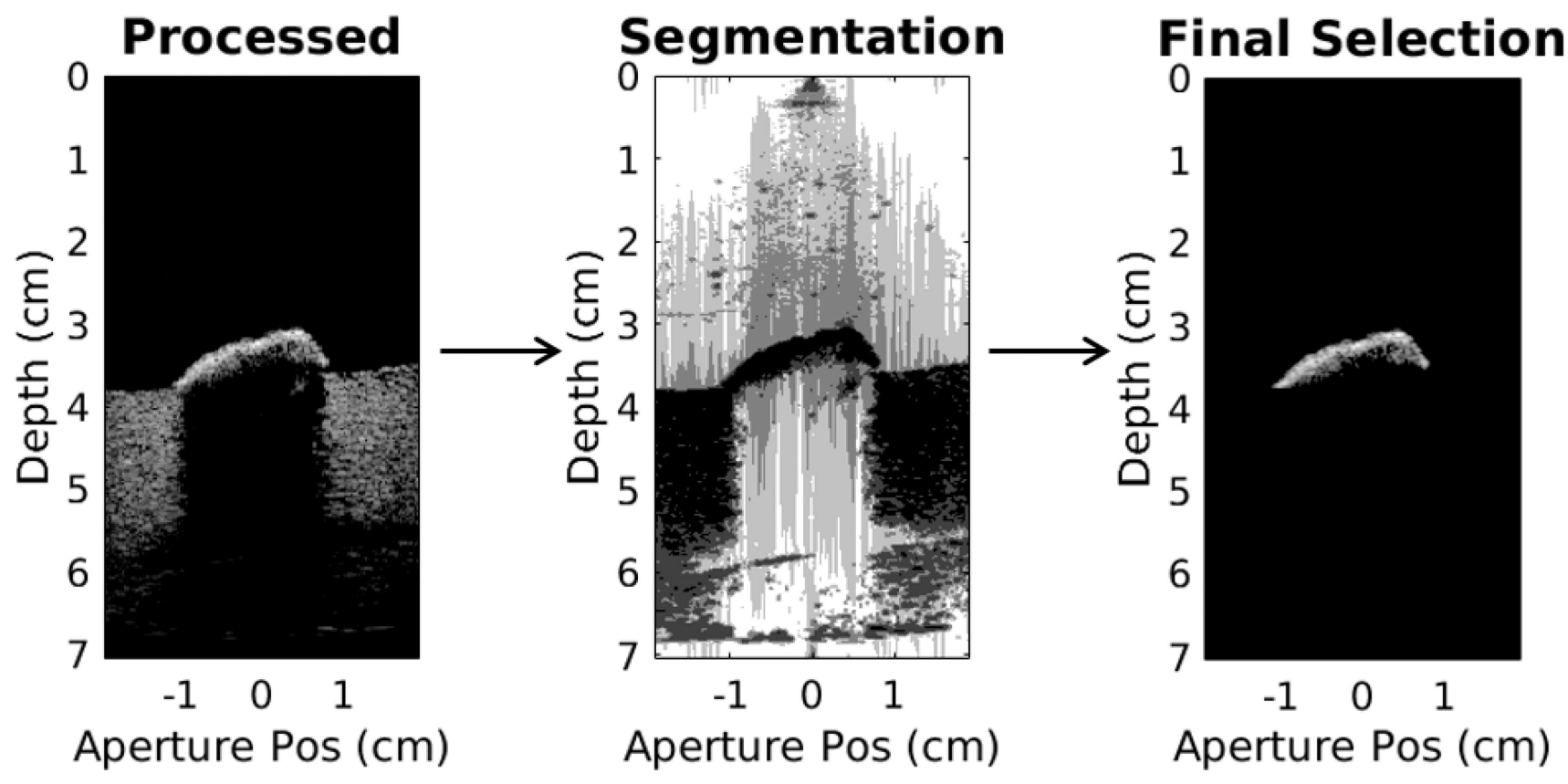

Figure 3.

Schematic of automated algorithm to isolate stone borders based on pixel intensity. 

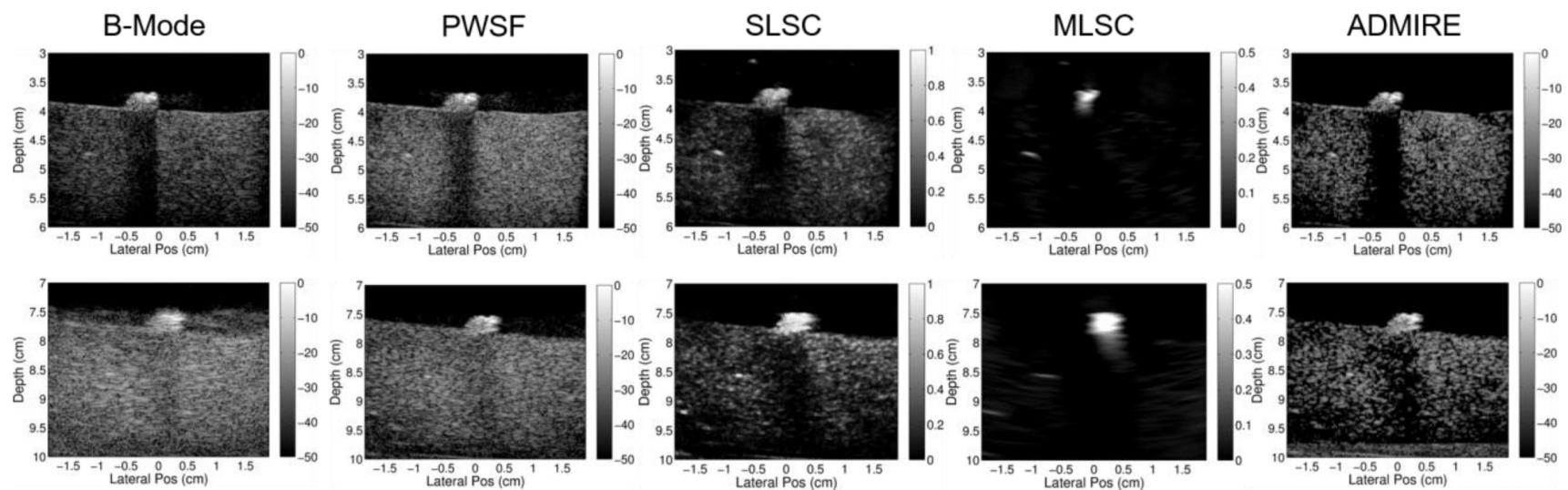

Figure 4.

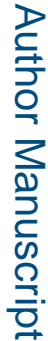

B-mode, PWSF, SLSC, MLSC, and ADMIRE images of a $5 \mathrm{~mm}$ stone at $4 \mathrm{~cm}$ (top) and $8 \mathrm{~cm}$ (bottom). 

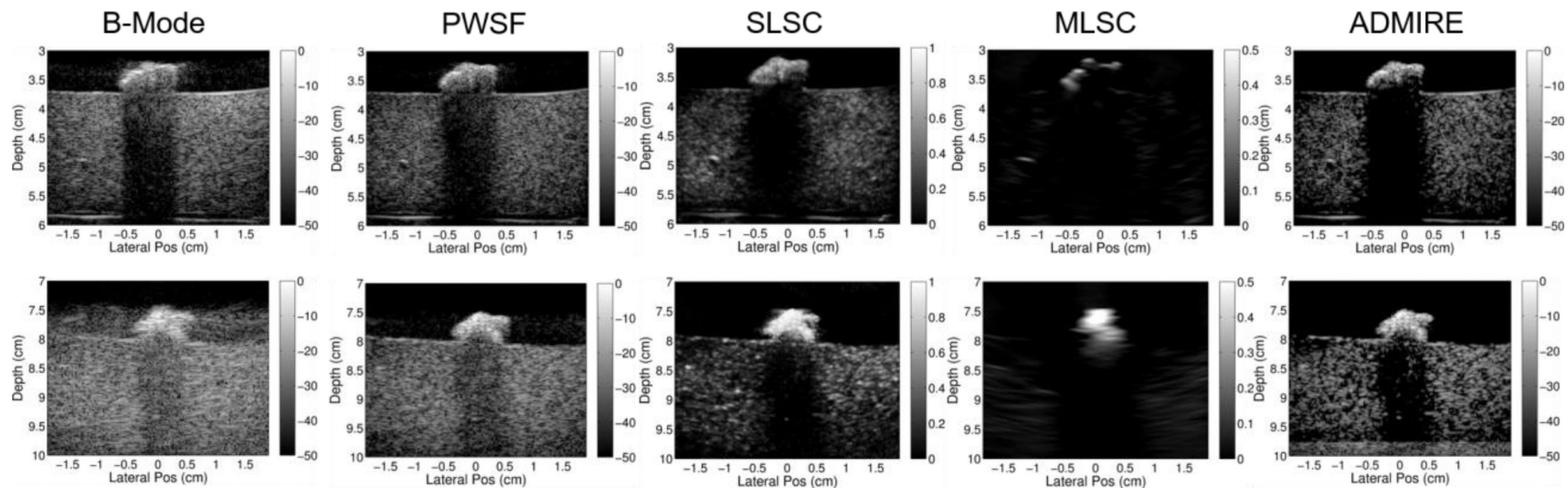

Figure 5.

B-mode, PWSF, SLSC, MLSC, and ADMIRE images of a 10mm stone at $4 \mathrm{~cm}$ (top) and $8 \mathrm{~cm}$ (bottom). Potential variability in the MLSC representation of the stone is visible in this pair located at $4 \mathrm{~cm}$ and $8 \mathrm{~cm}$. The MLSC image at $4 \mathrm{~cm}$ only shows the top surface of the stone, but the image at $8 \mathrm{~cm}$ provides a filled in representation of the stone. 


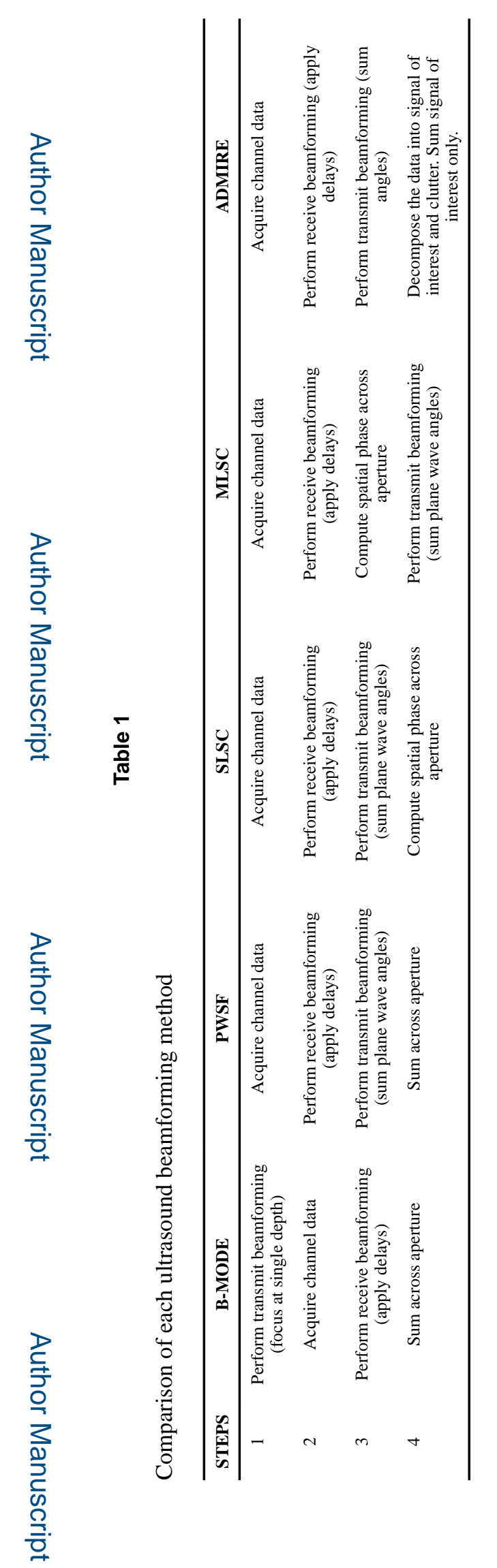

Urolithiasis. Author manuscript; available in PMC 2020 April 01. 


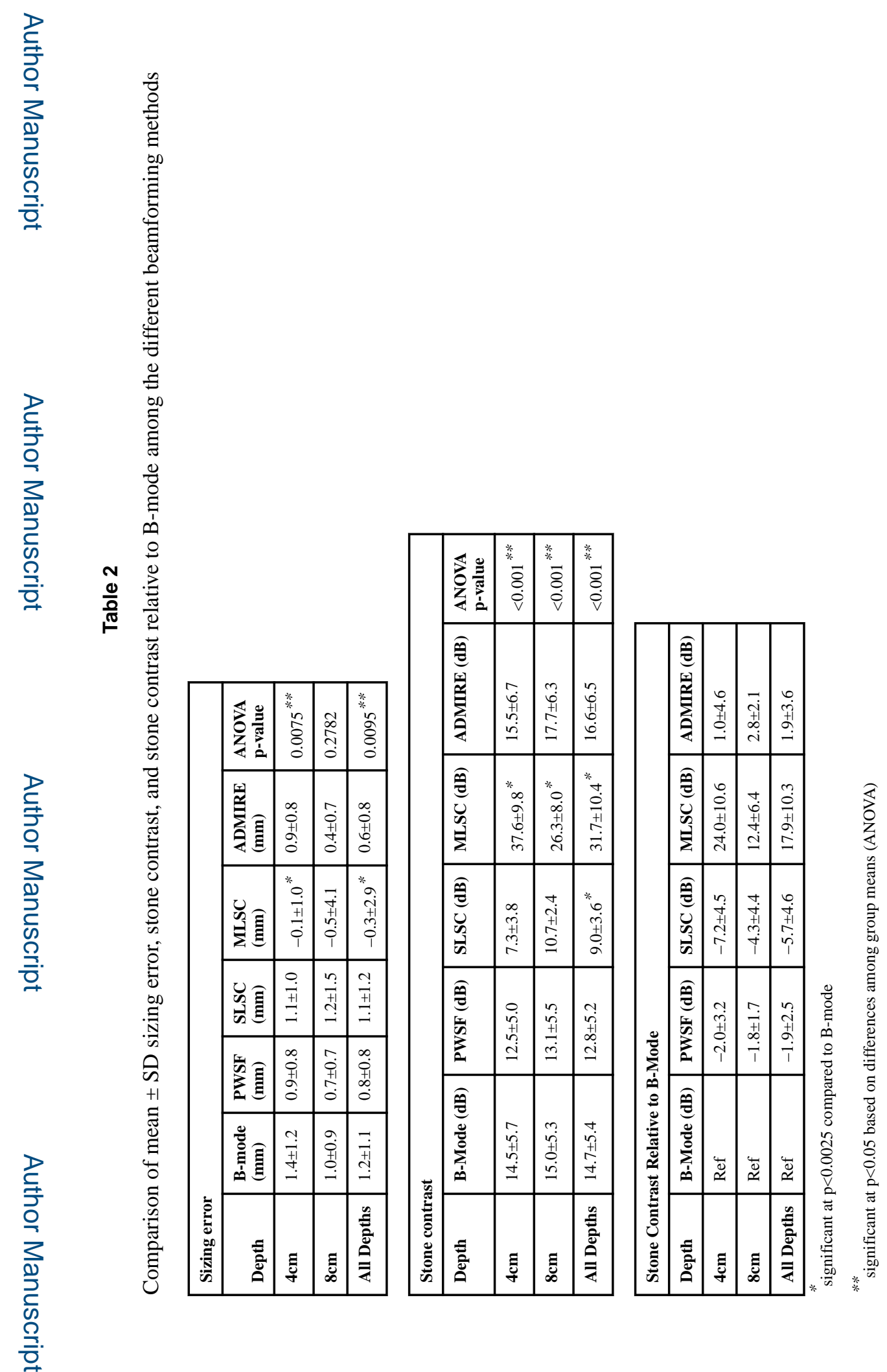

Urolithiasis. Author manuscript; available in PMC 2020 April 01. 